\title{
COMPOSITION OPERATORS ON ANALYTIC LIPSCHITZ SPACES
}

\author{
KEVIN M. MADIGAN
}

(Communicated by Palle E. T. Jorgensen)

\begin{abstract}
If $X$ is a Banach space of functions analytic on the disk and $\varphi: \mathrm{D} \rightarrow \mathrm{D}$ is analytic, one can define the composition operator $C_{\varphi}$ on $X$ by $C_{\varphi} f:=f \circ \varphi$. This paper discusses the boundedness and $w$-compactness of composition operators on the analytic Lipschitz spaces $\mathscr{A}_{\alpha}, 0<\alpha<1$.
\end{abstract}

\section{INTRODUCTION}

For $0<\alpha<1$, let $\mathscr{A}_{\alpha}$ be the space of functions $f$ analytic in the unit disk D of the complex plane that satisfy a Lipschitz condition of order $\alpha$; that is, $f \in \mathscr{A}_{\alpha}$ if and only if there is a constant $K>0$ such that $|f(z)-f(w)| \leq$ $K|z-w|^{\alpha}$ for all $z, w \in \mathbf{D}$. It is a well-known fact that when we equip $\mathscr{A}_{\alpha}$ with the norm

$$
\|f\|_{\alpha}=\sup \left\{|f(z)-f(w)| /|z-w|^{\alpha}: z, w \in \mathbf{D}, \quad z \neq w\right\},
$$

it is a Banach space (provided that we identify functions that differ by a constant). These spaces are very familiar to classical analysts. If $\varphi$ is an analytic function mapping $\mathbf{D}$ to $\mathbf{D}$, we can define a linear operator $C_{\varphi}$ on $\mathscr{A}_{\alpha}$, called a composition operator, by $C_{\varphi} f:=f \circ \varphi$. It is easy to see that an operator defined in this manner is linear. In this paper, the boundedness and $w$-compactness of such operators are studied. By the Closed Graph Theorem, $C_{\varphi}$ is bounded on $\mathscr{A}_{\alpha}$ if and only if $C_{\varphi}$ maps $\mathscr{A}_{\alpha}$ to $\mathscr{A}_{\alpha}$. The main results of this paper are Theorem A and Theorem B.

Theorem A. Let $0<\alpha<1$. Then $C_{\varphi}: \mathscr{A}_{\alpha} \rightarrow \mathscr{A}_{\alpha}$ is bounded if and only if

$$
\sup _{z \in \mathbf{D}}\left\{\left(\frac{1-|z|^{2}}{1-|\varphi(z)|^{2}}\right)^{1-\alpha}\left|\varphi^{\prime}(z)\right|\right\}<+\infty .
$$

It should be pointed out that there are functions in $\mathscr{A}_{\alpha}$ that do not satisfy the above condition.

Let $X$ be a Banach space. We say that a sequence $\left\{f_{n}\right\}$ in $X$ converges to $f$ weakly in $X$ if $\lim _{n \rightarrow \infty} L f_{n}=L f$ for every bounded linear functional $L$ on $X$. We say that a bounded linear operator $T: X \rightarrow X$ is $w$-compact if $\left\|T f_{n}\right\| \rightarrow 0$ as $n \rightarrow \infty$ whenever $f_{n} \rightarrow 0$ weakly in $X$. We say that $T$ is compact if the image of the closed unit ball in $X$ is compact in $X$.

Received by the editors February 10, 1992.

1991 Mathematics Subject Classification. Primary 47B38, 46E15; Secondary 47B07, 30C20. 
Theorem B. Let $0<\alpha<1$ and

$$
\lim _{|z| \rightarrow 1^{-}}\left(\frac{1-|z|^{2}}{1-|\varphi(z)|^{2}}\right)^{1-\alpha}\left|\varphi^{\prime}(z)\right|=0 .
$$

Then $C_{\varphi}: \mathscr{A}_{\alpha} \rightarrow \mathscr{A}_{\alpha}$ is $w$-compact; however, the $w$-compactness of $C_{\varphi}$ does not imply that this limit is 0 .

\section{Boundedness of $C_{\varphi}$}

Let $0<\alpha<1$, and define the metric $\rho_{\alpha}$ on $\mathbf{D}$ by $\rho_{\alpha}(z, w)=|z-w|^{\alpha}$. Then a function $f$, analytic in $\mathbf{D}$, is in $\mathscr{A}_{\alpha}$ if and only if there is a $K>0$ such that $|f(z)-f(w)| \leq K \rho_{\alpha}(z, w)$. This definition can easily be extended by replacing $\rho_{\alpha}$ with any metric on any domain. Suppose $\Omega$ is a domain in the complex plane $C$ and $d$ is a metric on $\Omega$. Define $X$ to be the space of functions analytic in $\Omega$ satisfying a Lipschitz type condition in $d$. In other words, a function $f$, analytic in $\Omega$, is in $X$ if and only if

$$
\sup \{|f(z)-f(w)| / d(z, w): z \neq w\}<+\infty .
$$

It is quite easy to see that if we define the norm

$$
\|f\|=\sup \{|f(z)-f(w)| / d(z, w): z \neq w\},
$$

then $X$ is a Banach space (provided that we identify functions that differ by a constant). We refer to a space defined in this manner as a Lipschitz type space on $\Omega$ induced by $d$.

Given a Banach space $X$ of analytic functions in $\Omega$, we can also define a function $d_{X}$ on $\Omega \times \Omega$ as follows:

$$
d_{X}(z, w)=\sup \left\{|f(z)-f(w)|:\|f\|_{X} \leq 1\right\} .
$$

$d_{X}$ is called the induced distance of the space $X$. Observe that $d_{X}$ is a distance on $\Omega$ if $X$ separates points of $\Omega$ and each point evaluation is a bounded linear functional on $X$. Note also that if $X$ is a Lipschitz type space induced by $d$, then, by definition, $d_{X}(z, w) \leq d(z, w)$ for all $z, w \in \Omega$ and equality holds if and only if $d$ is induced by a Banach space $Y$, not necessarily $X$. It is easy to show that $Y \subseteq X$ and equality holds if and only if $X$ is a Lipschitz type space; however, the distance that induces $X$ may not be $d_{X}$. It is possible that it is not even comparable to $d_{X}$, as we shall see below. The relationship between $X$ and its induced distance $d_{X}$ and between $d$ and its induced Lipschitz type space are studied in [Zhu2]. Now suppose $\varphi: \Omega \rightarrow \Omega$ is analytic, and define the operator $C_{\varphi}$ on $X$ by $C_{\varphi} f:=f \circ \varphi$. An operator defined in this manner is called a composition operator and is obviously linear. If $X$ and $Y$ are normed linear spaces, we say that a linear operator $T: X \rightarrow Y$ is bounded if $\|T\|=\sup \{\|T f\|:\|f\| \leq 1\}<+\infty$.

Lemma 1. Let $X$ be a Lipschitz type space on a domain $\Omega$ in $\mathbf{C}$, and let $\varphi: \Omega \rightarrow \Omega$. The composition operator $C_{\varphi}$ is bounded as an operator from $X$ to $X$ if and only if $d_{X}(\varphi(z), \varphi(w)) \leq K d_{X}(z, w)$ for some constant $K$ (depending on $\varphi$ ) and all $z, w \in \Omega$. 
Proof. First, assume $C_{\varphi}$ is bounded. Then we have

$$
\begin{aligned}
d_{X}(\varphi(z), \varphi(w)) & =\sup \{|f(\varphi(z))-f(\varphi(w))|:\|f\| \leq 1\} \\
& =\sup \left\{\left|C_{\varphi} f(z)-C_{\varphi} f(w)\right|:\|f\| \leq 1\right\} \\
& =\left\|C_{\varphi}\right\| \sup \left\{\left\|C_{\varphi}\right\|^{-1}\left|C_{\varphi} f(z)-C_{\varphi} f(w)\right|:\|f\| \leq 1\right\} \\
& \leq\left\|C_{\varphi}\right\| d_{X}(z, w) .
\end{aligned}
$$

Letting $K=\left\|C_{\varphi}\right\|$ gives the desired result.

Now, to prove the other direction, suppose $X$ is a Lipschitz type space induced by $d$, let $f \in X$, and observe that

$$
\begin{aligned}
\left|C_{\varphi} f(z)-C_{\varphi} f(w)\right| & =|f(\varphi(z))-f(\varphi(w))| \leq\|f\| d_{X}(\varphi(z), \varphi(w)) \\
& \leq K\|f\| d_{X}(z, w) \leq K\|f\| d(z, w)
\end{aligned}
$$

for all $z, w \in \Omega$. Therefore, for each $f \in X, C_{\varphi} f \in X$, with $\left\|C_{\varphi} f\right\| \leq K\|f\|$, and $C_{\varphi}$ is bounded with $\left\|C_{\varphi}\right\| \leq K$; the proof is completed.

The following lemma is a well-known result of Hardy and Littlewood and is stated without proof. See [Dur] and Theorem B in [DRS].

Lemma 2. Let $0<\alpha<1$. A function $f$, analytic in $\mathbf{D}$, belongs to the space $\mathscr{A}_{\alpha}$ if and only if $\sup \left\{\left(1-|z|^{2}\right)^{1-\alpha}\left|f^{\prime}(z)\right|: z \in \mathbf{D}\right\}$ is finite. Moreover, there is a constant $K_{\alpha}>0$ (depending only on $\alpha$ ) such that

$$
K_{\alpha}^{-1}\|f\|_{\alpha} \leq \sup \left\{\left(1-|z|^{2}\right)^{1-\alpha}\left|f^{\prime}(z)\right|: z \in \mathbf{D}\right\} \leq K_{\alpha}\|f\|_{\alpha}
$$

Now let $d_{\alpha}$ be the metric on $\mathbf{D}$ induced by $\mathscr{A}_{\alpha}$ with the norm \|\|$_{\alpha}$, and let

$$
d_{\alpha}^{\prime}(z, w)=\sup \left\{|f(z)-f(w)|: \sup \left\{\left(1-|z|^{2}\right)^{1-\alpha}\left|f^{\prime}(z)\right|: z \in \mathbf{D}\right\} \leq 1\right\} .
$$

This also quite clearly defines a metric on D. By Lemma 2, these metrics are comparable.

Lemma 3. For $0<\alpha<1$ and $z \in \mathbf{D}$ we have

$$
\lim _{w \rightarrow z} \frac{d_{\alpha}^{\prime}(z, w)}{|z-w|}=\left(1-|z|^{2}\right)^{\alpha-1} .
$$

Proof. See Theorem 17 in [Zhu1].

In [Zhu2] it is shown that for any Banach space $X$ of analytic functions on a domain $\Omega \subset \mathbf{C}$,

$$
\lim _{w \rightarrow z} \frac{d_{X}(z, w)}{|z-w|}=\sup \left\{\left|f^{\prime}(z)\right|:\|f\|_{X} \leq 1\right\}
$$

for all $z \in \Omega$. This fact, together with Lemmas 2 and 3, tells us that

$$
K_{\alpha}^{-1}\left(1-|z|^{2}\right)^{\alpha-1} \leq \lim _{w \rightarrow z} \frac{d_{\alpha}(z, w)}{|z-w|} \leq K_{\alpha}\left(1-|z|^{2}\right)^{\alpha-1},
$$

where $K_{\alpha}$ is a positive constant depending only on $\alpha$. Lemma 3 also shows us that $\rho_{\alpha}$ and $d_{\alpha}$ are not comparable, but we know that they induce the same Lipschitz type space. The interesting reader is referred to [Zhu1, Zhu2]. 
Theorem 4. Let $0<\alpha<1$ and $\varphi: \mathbf{D} \rightarrow \mathbf{D}$ be analytic. Then the operator $C_{\varphi}: \mathscr{A}_{\alpha} \rightarrow \mathscr{A}_{\alpha}$ is bounded if and only if

$$
\sup _{z \in \mathbf{D}}\left\{\left(\frac{1-|z|^{2}}{1-|\varphi(z)|^{2}}\right)^{1-\alpha}\left|\varphi^{\prime}(z)\right|\right\}<+\infty .
$$

Proof. First, let $f \in \mathscr{A}_{\alpha}$ and assume (1) is satisfied. Then by Lemma 2 there is a positive constant $K_{\alpha}$ (depending only on $\alpha$ ) such that

$$
\begin{aligned}
\left(1-|z|^{2}\right)^{1-\alpha}\left|\left(C_{\varphi} f\right)^{\prime}(z)\right| & =\left(1-|z|^{2}\right)^{1-\alpha}\left|f^{\prime}(\varphi(z)) \| \varphi^{\prime}(z)\right| \\
& \leq K_{\alpha}\|f\|_{\alpha}\left(\frac{1-|z|^{2}}{1-|\varphi(z)|^{2}}\right)^{1-\alpha}\left|\varphi^{\prime}(z)\right|
\end{aligned}
$$

for all $z \in \mathbf{D}$. Hence, by Lemma 2 again, $\left\|C_{\varphi} f\right\|_{\alpha} \leq M\|f\|_{\alpha}$, where

$$
M=K_{\alpha}^{2} \sup _{z \in \mathbf{D}}\left\{\left(\frac{1-|z|^{2}}{1-|\varphi(z)|^{2}}\right)^{1-\alpha}\left|\varphi^{\prime}(z)\right|\right\}
$$

and $C_{\varphi}$ is bounded.

To prove the other direction, assume $C_{\varphi}$ is bounded. By Lemma 1, $d_{\alpha}(\varphi(z), \varphi(w)) \leq K d_{\alpha}(z, w)$ for some constant $K$ (depending on $\varphi$ ) and all $z, w \in \mathbf{D}$. So

$$
K \geq \frac{d_{\alpha}(\varphi(z), \varphi(w))}{d_{\alpha}(z, w)}=\frac{d_{\alpha}(\varphi(z), \varphi(w))}{|\varphi(z)-\varphi(w)|} \frac{|\varphi(z)-\varphi(w)|}{|z-w|} \frac{|z-w|}{d_{\alpha}(z, w)}
$$

and

$$
\limsup _{w \rightarrow z} \frac{d_{\alpha}(\varphi(z), \varphi(w))}{|\varphi(z)-\varphi(w)|} \frac{|\varphi(z)-\varphi(w)|}{|z-w|} \frac{|z-w|}{d_{\alpha}(z, w)} \leq K .
$$

But, by the remark after Lemma 3 and the analyticity of $\varphi$, this limit exists and is comparable to

$$
\left(\frac{1-|z|^{2}}{1-|\varphi(z)|^{2}}\right)^{1-\alpha}\left|\varphi^{\prime}(z)\right|
$$

hence,

$$
\sup _{z \in \mathbf{D}}\left\{\left(\frac{1-|z|^{2}}{1-|\varphi(z)|^{2}}\right)^{1-\alpha}\left|\varphi^{\prime}(z)\right|\right\}<+\infty,
$$

and we are done.

Corollary 5. Let $0<\alpha<1$. If $C_{\varphi}: \mathscr{A}_{\alpha} \rightarrow \mathscr{A}_{\alpha}$ is bounded and $\lim _{r \rightarrow 1^{-}}\left|\varphi\left(r e^{i \theta}\right)\right|$ $=1$ for some $\theta \in[0,2 \pi)$ then $\lim _{r \rightarrow 1^{-}}\left(1-r^{2}\right)^{1-\alpha}\left|\varphi^{\prime}\left(r e^{i \theta}\right)\right|=0$.

Proof. Since $C_{\varphi}$ is bounded, there is a constant $M>0$ such that $\left(1-|z|^{2}\right)^{1-\alpha}\left|\varphi^{\prime}(z)\right| \leq M\left(1-|\varphi(z)|^{2}\right)^{1-\alpha}$ for all $z \in \mathbf{D}$. The desired result easily follows.

At this point it would be quite natural for one to ask if, for $0<\alpha<1$, there is a $\varphi \in \mathscr{A}_{\alpha}$ with $|\varphi(z)|<1$ for all $z \in \mathbf{D}$ such that $C_{\varphi}$ is not bounded from $\mathscr{A}_{\alpha}$ to $\mathscr{A}_{\alpha}$. The answer to this question is that there are, in fact, many such functions. In order to show this we will make use of some classical results about conformal mappings from the disk to polygons. We use the same notation that is used in [Ah1], and most of the remainder of this paragraph is quoted from 
there. Let $P$ be an $n$-gon, and let $\Omega$ be the region bounded by $P$. Let the consecutive vertices be $z_{1}, \ldots, z_{n}$ in positive cyclic order (we set $z_{n+1}=z_{1}$ ). The angle at $z_{k}$ is defined to be $\arg \left(\left(z_{k-1}-z_{k}\right) /\left(z_{k+1}-z_{k}\right)\right)$ between 0 and $2 \pi$. We shall denote it by $a_{k} \pi, 0<a_{k}<2$. It is also convenient to introduce the outer angles $\beta_{k} \pi=\left(1-a_{k}\right) \pi,-1<\beta_{k}<1$. It can easily be shown that $\beta_{1}+\cdots+\beta_{n}=2$ and that $P$ is convex if and only if all $\beta_{k}>0$. All polygons in the remainder of this section are assumed to be convex.

By the Riemann Mapping Theorem there are conformal maps $F: \mathrm{D} \rightarrow \Omega$, which can be extended homeomorphically to map $\overline{\mathbf{D}}$ to $\bar{\Omega}$, mapping points $w_{k} \in \partial \mathbf{D}$ to the vertices $z_{k}$ of $P$ and mapping the arcs between consecutive $w_{k}$ 's to the sides of $P$. By the Schwarz-Christoffel formula, any such $F$ can be written as

$$
F(z)=C \int_{0}^{z} \prod_{k=1}^{n}\left(w-w_{k}\right)^{-\beta_{k}} d w+C^{\prime},
$$

where $C$ and $C^{\prime}$ are complex constants. Both of the above theorems are classical and can be found in many standard complex analysis texts, including [Ah1].

Lemma 6. Let $0<\alpha<1$, let $n$ be a positive integer, and let $P \subset \overline{\mathbf{D}}$ be an $n$-gon with angles $a_{k} \pi(k=1, \ldots, n)$. Let $\Omega$ be the region bounded by $P$, and let $F: \mathbf{D} \rightarrow \Omega$ be a conformal mapping given by the Schwarz-Christoffel formula. Then $F \in \mathscr{A}_{\alpha}$ if and only if $a_{k} \geq \alpha(k=1, \ldots, n)$.

Proof. We use the characterization of $\mathscr{A}_{\alpha}$ given by Lemma 2 :

$$
\sup _{z \in \mathbf{D}}\left\{\left(1+|z|^{2}\right)^{1-\alpha}\left|F^{\prime}(z)\right|\right\}=|C| \sup _{z \in \mathbf{D}}\left\{\left(1-|z|^{2}\right)^{1-\alpha} \prod_{k=1}^{n}\left|z-w_{k}\right|^{-\beta_{k}}\right\},
$$

which is finite if and only if $1-\alpha \geq \beta_{k}(k=1, \ldots, n)$. Since $\beta_{k}=1-a_{k}$, we are done.

Since $\beta_{1}+\cdots+\beta_{n}=2$, Lemma 6 tells us that $F \in \mathscr{A}_{\alpha}$ implies $\alpha \leq 1-2 / n$.

Corollary 7. Let $0<\alpha<1$, let $n$ be a positive integer, and let $P \subset \overline{\mathbf{D}}$ be a regular $n$-gon. Let $\Omega$ be the region bounded by $P$, and let $F: \mathbf{D} \rightarrow \Omega$ be a conformal mapping given by the Schwarz-Christoffel formula. Then $F \in \mathscr{A}_{\alpha}$ if and only if $\alpha \leq 1-2 / n$.

Proof. By Lemma 6, $F \in \mathscr{A}_{\alpha}$ if and only if $\alpha \leq 1-\beta_{k}(k=1, \ldots, n)$. Since $P$ is regular, each $\beta_{k}=2 / n$, giving us the desired result.

Theorem 8. Let $0<\alpha<1$. There exists $F \in \mathscr{A}_{\alpha}$ with $|F(z)|<1$ for all $z \in \mathbf{D}$ such that $C_{F}$ is not bounded from $\mathscr{A}_{\alpha}$ to $\mathscr{A}_{\alpha}$.

Proof. The proof is constructive. Choose a positive integer $n$ such that $\alpha \leq$ $1-2 / n$, and let $P \subset \overline{\mathbf{D}}$ be an $n$-gon with angles $a_{k} \pi(k=1, \ldots, n)$ such that one of the vertices, say $z_{1}$, is on the unit circle. Let us also require that $a_{1}=\alpha$ and, for $k \neq 1, a_{k} \geq \alpha$. Let $\Omega$ be the region bounded by $P$, and let $F: \mathrm{D} \rightarrow \Omega$ be a conformal mapping given by the Schwarz-Christoffel formula. $F \in \mathscr{A}_{\alpha}$ due to Lemma 6. Let $\theta=\arg \left(w_{1}\right)$, and notice that $\beta_{1}=1-\alpha$. Then

$$
\lim _{r \rightarrow 1^{-}}\left|F\left(r e^{i \theta}\right)\right|=\left|z_{1}\right|=1
$$


but

$$
\begin{aligned}
\lim _{r \rightarrow 1^{-}}\left(1-r^{2}\right)^{1-\alpha}\left|F^{\prime}\left(r e^{i \theta}\right)\right| & =|C| \prod_{k=2}^{n}\left|e^{i \theta}-w_{k}\right|^{-\beta_{k}} \lim _{r \rightarrow 1^{-}} \frac{\left(1-r^{2}\right)^{1-\alpha}}{\left|r e^{i \theta}-w_{1}\right|^{\beta_{1}}} \\
& =2^{1-\alpha}|C| \prod_{k=2}^{n}\left|e^{i \theta}-w_{k}\right|^{-\beta_{k}} \neq 0 .
\end{aligned}
$$

So by Corollary 5, $C_{F}$ is not bounded from $\mathscr{A}_{\alpha}$ to $\mathscr{A}_{\alpha}$.

\section{3. $W$-COMPACTNESS OF $C_{\varphi}$}

Recall that a linear operator $T$ defined on a Banach space $X$ is said to be $w$-compact if $\left\|T f_{n}\right\| \rightarrow 0$ whenever $f_{n} \rightarrow 0$ weakly in $X$. It is easy to show that $w$-compact operators are bounded. If $X$ is a Banach space and $T$ is compact, then $T$ is $w$-compact, and if $X$ is reflexive, then $T$ is compact if and only if it is $w$-compact; see [Rud].

Proposition 9. Let $0<\alpha<1$. If $f_{n} \rightarrow 0$ weakly in $\mathscr{A}_{\alpha}$, then $\left\|f_{n}\right\|_{\alpha}$ are bounded and $f_{n}^{\prime}(z) \rightarrow 0$ uniformly on compact subsets of $\mathbf{D}$.

Proof. The uniform boundedness principle guarantees the boundedness of the norms of the sequence elements. Now let $\mathbf{C}$ be a compact subset of $\mathbf{D}$. There exists an $r \in(0,1)$ such that $|z| \leq r$ for all $z \in \mathbf{C}$. Let $z \in \mathbf{C}$. Then by Lemma 2

$$
\left|f_{n}^{\prime}(z)\right| \leq \frac{K_{\alpha}\left\|f_{n}\right\|_{\alpha}}{\left(1-|z|^{2}\right)^{1-\alpha}} \leq \frac{K_{\alpha} M}{\left(1-r^{2}\right)^{1-\alpha}},
$$

for some constant $M>0$. Hence $\left\{f_{n}^{\prime}\right\}$ is uniformly bounded on compact subsets of $\mathbf{D}$, which implies that it is a normal family. For $z \in \mathbf{D}$, define $T_{z} f=f^{\prime}(z)$. Since

$$
\left|f^{\prime}(z)\right| \leq \frac{K_{\alpha}\|f\|_{\alpha}}{\left(1-|z|^{2}\right)^{1-\alpha}}
$$

$T_{z}$ is a bounded linear functional on $\mathscr{A}_{\alpha}$. Therefore, $T_{z} f_{n}=f_{n}^{\prime}(z) \rightarrow 0$ as $n \rightarrow \infty$, and this is true for all $z \in \mathbf{D}$. This, taken together with the fact that there is a subsequence $\left\{f_{n_{k}}\right\}$ that converges uniformly on compact subsets of D, finishes the proof.

Proposition 10. If $\varphi \in \mathscr{A}_{\alpha}$ and $\overline{\varphi(\mathbf{D})} \subset \mathbf{D}$ is compact, then $C_{\varphi}$ is w-compact from $\mathscr{A}_{\alpha} \rightarrow \mathscr{A}_{\alpha}$.

Proof. Suppose $f_{n} \rightarrow 0$ weakly in $\mathscr{A}_{\alpha}$. By Lemma 2

$$
\left\|C_{\varphi} f_{n}\right\|_{\alpha} \leq K_{\alpha} \sup _{z \in \mathbf{D}}\left\{\left(1-|z|^{2}\right)^{1-\alpha}\left|f_{n}^{\prime}(\varphi(z)) \| \varphi^{\prime}(z)\right|\right\} \leq\|\varphi\|_{\alpha} \sup _{z \in \mathbf{D}}\left\{\left|f_{n}^{\prime}(\varphi(z))\right|\right\} .
$$

Since $\overline{\varphi(\mathbf{D})}$ is a compact subset of $\mathbf{D}$ and $f_{n}^{\prime}(z) \rightarrow 0$ uniformly on compact subsets of $\mathbf{D}$,

$$
\left\|C_{\varphi} f_{n}\right\|_{\alpha} \leq\|\varphi\|_{\alpha} \sup _{w \in \varphi(\mathbf{D})}\left|f_{n}^{\prime}(w)\right| \rightarrow 0
$$

as $n \rightarrow \infty$ and $C_{\varphi}$ is $w$-compact.

Lemma 11. Let $\varphi$ be in $\mathscr{A}_{\alpha}$ such that $|\varphi(z)| \leq 1$ for all $z$ in $\mathbf{D}$, and let $C$ be a compact subset of $\mathbf{D}$. Then for each $\varepsilon>0$ there is $a \delta>0$ such that if $1-\delta<r<1$ then $\left|f^{\prime}(\varphi(r z)) \varphi^{\prime}(r z) r-f^{\prime}(\varphi(z)) \varphi^{\prime}(z)\right|<\varepsilon$ for all $z \in C$ and $f \in \mathscr{A}_{\alpha}$ with $\|f\|_{\alpha} \leq 1$. 
Proof. We will make use of the Cauchy integral formula for the derivative of an analytic function. Since $C$ is compact, there is a $\rho \in(0,1)$ such that for each $z \in C,|z| \leq \rho$. Let $R=\rho+(1-\rho) / 2$, and let $C_{R}$ denote the circle of radius $R$. Let $z \in C$ and $\|f\|_{\alpha} \leq 1$. Then

$$
\begin{aligned}
& \left|f^{\prime}(\varphi(r z)) \varphi^{\prime}(r z) r-f^{\prime}(\varphi(z)) \varphi^{\prime}(z)\right| \\
& \quad \leq \frac{1}{2 \pi} \int_{C_{R}} \frac{|f(\varphi(r w))-f(\varphi(w))|}{|w-z|^{2}}|d w| \leq \frac{1}{2 \pi} \int_{C_{R}} \frac{|\varphi(r w)-\varphi(w)|^{\alpha}}{|w-z|^{2}}|d w| \\
& \quad \leq \frac{\|\varphi\|_{\alpha}^{\alpha}}{2 \pi} \int_{C_{R}} \frac{|r w-w|^{\alpha^{2}}}{|w-z|^{2}}|d w| \leq \frac{4\|\varphi\|_{\alpha}^{\alpha}(1-r)^{\alpha^{2}}}{(1-\rho)^{2}} .
\end{aligned}
$$

This last quantity tends to 0 as $r$ tends to 1 , which completes the proof.

Theorem 12. Let $0<\alpha<1, \varphi: \mathbf{D} \rightarrow \mathbf{D}$ be analytic such that $C_{\varphi}: \mathscr{A}_{\alpha} \rightarrow \mathscr{A}_{\alpha}$ is bounded, and

$$
\lim _{|z| \rightarrow 1^{-}}\left(\frac{1-|z|^{2}}{1-|\varphi(z)|^{2}}\right)^{1-\alpha}\left|\varphi^{\prime}(z)\right|=0 .
$$

Then $C_{\varphi}: \mathscr{A}_{\alpha} \rightarrow \mathscr{A}_{\alpha}$ is $w$-compact.

Proof. For $0<r<1$, define $\varphi_{r}(z)=\varphi(r z) . \overline{\varphi_{r}(\mathbf{D})}$ is a compact subset of $\mathbf{D}$, so by Proposition $10, C_{\varphi_{r}}: \mathscr{A}_{\alpha} \rightarrow \mathscr{A}_{\alpha}$ is $w$-compact. Assume that $\lim _{r \rightarrow 1^{-}}\left\|C_{\varphi_{r}}-C_{\varphi}\right\|=0$ and that $f_{n} \rightarrow 0$ weakly in $\mathscr{A}_{\alpha}$. We know there is a constant $M>0$ such that $\left\|f_{n}\right\| \leq M$ for all $n$. Let $\varepsilon>0$.

$$
\begin{aligned}
\left\|C_{\varphi} f_{n}\right\| & \leq\left\|C_{\varphi_{r}} f_{n}-C_{\varphi} f_{n}\right\|+\left\|C_{\varphi_{r}} f_{n}\right\| \\
& \leq\left\|f_{n}\right\|\left\|C_{\varphi_{r}}-C_{\varphi}\right\|+\left\|C_{\varphi_{r}} f_{n}\right\| \\
& \leq M\left\|C_{\varphi_{r}}-C_{\varphi}\right\|+\left\|C_{\varphi_{r}} f_{n}\right\| .
\end{aligned}
$$

Choose $r \in(0,1)$ such that $\left\|C_{\varphi_{r}}-C_{\varphi}\right\|<\varepsilon / 2 M$, and choose $n_{0} \in \mathbf{N}$ such that $\left\|C_{\varphi_{r}} f_{n}\right\|<\varepsilon / 2$ for $n>n_{0}$. Then $\left\|C_{\varphi} f_{n}\right\|<\varepsilon$ and $C_{\varphi}$ is $w$-compact.

All that remains is to show that $\lim _{r \rightarrow 1^{-}}\left\|C_{\varphi_{r}}-C_{\varphi}\right\|=0$ whenever (2) holds. Let $\varepsilon>0$ and choose $\rho \in(0,1)$ such that $\rho \leq|z|<1$ implies that

$$
\left(\frac{1-|z|^{2}}{1-|\varphi(z)|^{2}}\right)^{1-\alpha}\left|\varphi^{\prime}(z)\right|<\frac{\varepsilon}{4}
$$

Let $R \in(\rho, 1)$ and choose $\delta \in(0,1)$ such that $1-\delta<r<1$ implies that

$$
\left|f^{\prime}(\varphi(z)) \varphi^{\prime}(z)-r f^{\prime}(\varphi(r z)) \varphi^{\prime}(r z)\right|<\varepsilon / 2
$$

for $|z| \leq R$ and $\|f\|_{\alpha} \leq 1$ (Lemma 11 guarantees that we can do this). Let $r \in(a, 1)$, where $a=\max \left\{1-\delta, \frac{\rho}{R}\right\}$. Notice that $\rho / r<R$. Applying Lemma 2, we have

$$
\begin{aligned}
\left\|C_{\varphi_{r}}-C_{\varphi}\right\| & \leq K_{\alpha} \sup _{\|f\|_{\alpha} \leq 1}\left\{\sup _{z \in \mathbf{D}}\left\{\left(1-|z|^{2}\right)^{1-\alpha}\left|f^{\prime}(\varphi(z)) \varphi^{\prime}(z)-r f^{\prime}(\varphi(r z)) \varphi^{\prime}(r z)\right|\right\}\right\} \\
& \leq K_{\alpha}(A+B)
\end{aligned}
$$

where

$$
A=\sup _{\|f\|_{\alpha} \leq 1}\left\{\sup _{|z| \leq \rho / r}\left\{\left(1-|z|^{2}\right)^{1-\alpha}\left|f^{\prime}(\varphi(z)) \varphi^{\prime}(z)-r f^{\prime}(\varphi(r z)) \varphi^{\prime}(r z)\right|\right\}\right\}
$$


and

$$
B=\sup _{\|f\|_{\alpha} \leq 1}\left\{\sup _{\rho / r<|z|<1}\left\{\left(1-|z|^{2}\right)^{1-\alpha}\left|f^{\prime}(\varphi(z)) \varphi^{\prime}(z)-r f^{\prime}(\varphi(r z)) \varphi^{\prime}(r z)\right|\right\}\right\} .
$$

We have chosen our parameters so that $\rho / r<R$ and $r>1-\delta$, so $A<\varepsilon / 2$. Let us now concentrate on $B$. Since $\left(1-|z|^{2}\right)^{1-\alpha}<\left(1-|r z|^{2}\right)^{1-\alpha}, B \leq$ $\sup _{\|f\|_{\alpha} \leq 1}\left\{B_{1}+B_{2}\right\}$, where

$$
B_{1}=\sup _{\rho / r<|z|<1}\left\{\left(\frac{1-|z|^{2}}{1-|\varphi(z)|^{2}}\right)^{1-\alpha}\left|\varphi^{\prime}(z) \| f^{\prime}(\varphi(z))\right|\left(1-|\varphi(z)|^{2}\right)^{1-\alpha}\right\}
$$

and

$$
B_{2}=\sup _{\rho / r<|z|<1}\left\{\left(\frac{1-|r z|^{2}}{1-|\varphi(r z)|^{2}}\right)^{1-\alpha}\left|\varphi^{\prime}(r z) \| f^{\prime}(\varphi(r z))\right|\left(1-|\varphi(r z)|^{2}\right)^{1-\alpha}\right\} .
$$

We also have $|z|>|r z|>\rho$, so $B_{1} \leq K_{\alpha}\|f\|_{\alpha} \varepsilon / 4$. If we replace $B_{1}$ with $B_{2}$ in the above inequality, it will still hold. We chose $f$ so that $\|f\|_{\alpha} \leq 1$, and so $\sup _{\|f\|_{\alpha} \leq 1}\left\{B_{1}+B_{2}\right\} \leq K_{\alpha} \varepsilon / 2$. This gives us our desired result, i.e., (2) implies that $\lim _{r \rightarrow 1^{-}}\left\|C_{\varphi_{r}}-C_{\varphi}\right\|=0$, and $C_{\varphi}$ is $w$-compact.

Theorem 13. Let $0<\alpha<1$. There exists an analytic function $f: \mathbf{D} \rightarrow \mathbf{D}$ with $C_{f} w$-compact on $\mathscr{A}_{\alpha}$ that does not satisfy condition (2).

Proof. Let $f(z)=4^{-\alpha}(1-z)^{\alpha}$, choosing the branch where $f(0)=4^{-\alpha}$. Notice that $f(1)=0$. Clearly, $f$ is analytic in $\mathbf{D}$, and $|f(z)| \leq 2^{-\alpha}$ for all $z \in \mathbf{D}$, so that $\overline{f(\mathbf{D})}$ is a compact subset of $\mathbf{D}$. So, by Proposition 10, $C_{f}$ is $w$-compact; however,

$$
\lim _{r \rightarrow 1^{-}}\left(\frac{1-r^{2}}{1-|f(r)|^{2}}\right)^{1-\alpha}\left|f^{\prime}(r)\right|=\lim _{r \rightarrow 1^{-}}\left(1-r^{2}\right)^{1-\alpha} \alpha(1-r)^{\alpha-1}=2^{1-\alpha} \alpha .
$$

Therefore $f$ does not satisfy condition (2).

\section{ADDED IN PROOF}

After this paper was submitted, two other papers were brought to the attention of the author. N. Zorboska pointed out that boundedness and compactness of $C_{\varphi}$ on $\mathscr{A}_{\alpha}$ has been studied in [Roan]. Additionally, one of the referees for this paper observed that a corollary in [Roan] proves one direction of Theorem 4 , and that there are examples in [Roan] which are relevant to Theorem 8 . The referee also pointed out [Shap], wherein it is shown that compact composition operators on a wide variety of spaces of holomorphic functions contained in the disk algebra, including the $\mathscr{A}_{\alpha}$ spaces, must satisfy $\|\varphi\|_{\infty}<1$. Thus, a complete characterization of both the bounded and compact $C_{\varphi}$ on $\mathscr{A}_{\alpha}$ is already known. However, my statements of results, ideas, and methods of proof are different from those in both [Roan] and [Shap]. 


\section{ACKNOWLEDGMENT}

The author would like to thank his thesis advisor, Kehe Zhu, for very helpful conversations on this topic and advice in preparing this manuscript.

\section{REFERENCES}

[Ah1] L. Ahlfors, Complex analysis, McGraw-Hill, New York, 1979.

[DRS] P. Duren, B. Romberg, and A. Shields, Linear functionals on $H^{p}$ spaces with $0<p<1$, J. Reine Angew. Math. 238 (1969), 32-60.

[Dur] P. L. Duren, Theory of $H^{p}$ spaces, Academic Press, Boston, MA, 1970.

[Roan] R. Roan, Composition operators on a space of Lipschitz functions, Rocky Mountain J. Math. 10 (1980), 371-379.

[Rud] W. Rudin, Functional analysis, 2nd ed., McGraw-Hill, New York, 1991.

[Shap] J. Shapiro, Compact composition operators on spaces of boundary regular holomorphic functions, Proc. Amer. Math. Soc. 100 (1987), 49-57.

[Zhu1] K. Zhu, Bloch type spaces of analytic functions, Rocky Mountain J. Math. (to appear).

[Zhu2] __ Distances and Banach spaces of holomorphic functions on complex domains, J. London Math. Soc. (to appear).

Department of Mathematics and Statistics, State University of New York at Albany, AlbaNy, New York 12222

E-mail address: km9985@math.albany.edu 